(NASA-CR-200033) COMPLETING THE COPERNICAN REVOLUTION: THE SEARCH

FOR OTHER PLANETARY SYSTEMS (Lunar and Planetary Inst.) $22 \mathrm{p}$
N96-17146

Unclas

$63 / 91 \quad 0092357$ 


\title{
COMPLETING THE COPERNICAN REVOLUTION: The Search for Other Planetary Systems
}

\author{
David C. Black \\ Lunar and Planetary Institute, Houston, Texas 77058
}

KEY WORDS: astrometry, radial velocity, brown dwarfs, pulsars

\begin{abstract}
The past few decades have witnessed significant advances in our understanding of how stars form, and there has been an associated increase in our knowledge of conditions and phenomena in the early solar system. These have led to the formulation of a paradigm for the origin of the solar system that is sufficiently complete that its basic elements can be tested directly through observations. A simple, but profound, consequence of the paradigm is that most if not all stars should be accompanied by planetary systems. The accuracy of instruments that can be used in such searches has improved to the point that Jupiter-like companions to a number of nearby stars could be detected. However, the results to date are that no other planetary systems have been detected, and the absence of detection is becoming statistically significant, particularly as it relates to the existence of brown dwarf companions to main-sequence stars.
\end{abstract}

\section{INTRODUCTION}

Accounts vary as to when humans first observed the heavens in any systematic fashion. In our ancestors' earliest stages of evolution as hunter-gathers, did they associate the migration of prey with the appearance or passing of groupings of stars? Did the discovery of agriculture and the need to understand seasons herald the dawn of astronomy? It is clear that by the time of the golden age of Greece, astronomers were well aware of and knowledgeable about the heavens. They recognized that among the dwellers of the night sky there were a small number of objects that displayed extensive, varied, and highly regular motion 
with respect to the other denizens of the sky. These were called "planetes," the Greek word for wanderers that serves as the root for the word "planets."

Efforts to understand the motion of celestial objects were the focus of much of astronomy from the time of the Greeks until the middle of the second millennium AD. Those efforts were generally based on the assumption that the Earth was the center of the universe and that all of the motions displayed by objects in the heavens were with reference to the Earth. While models could be developed to explain the observed motion, they were complex and grew more so as more refined observations became available.

The situation changed in the 1500 s when the Polish astronomer Nikolas Copernicus challenged the notion that the Earth was the center of the universe, asserting that the Earth along with the other planets revolved around the Sun. This shift in perspective for the motion of the heavens had great implications for many aspects of human thought, not the least of which was the removal of mankind from the center of the universe. This time in history is often referred to as the beginning of the Copernican Revolution.

It is fitting that continuation of the Copernican Revolution was also driven by astronomy with the advent of bigger and better telescopes and with increasingly sensitive detectors. We now know that the Sun is located in the outer reaches of the Galaxy and is an otherwise unexceptional star in comparison with the roughly one hundred billion stars in the Galaxy. We also know that the Galaxy is but one among many tens to hundreds of billions of galaxies. All of this suggests that the Solar System is not unique in the universe, nor for that matter is life, even intelligent life. However, in spite of the many advances in observational astronomy since the time of Copernicus, the nonuniqueness of the Solar System and life remains only a suggestion.

We have at the present time no confirmed observational evidence either for another planetary system or for extraterrestrial life.

This article deals with the former-searches for other planetary systems. The outcome of these searches will bring us close to completing the revolution in thought started by Copernicus some 500 years ago and in so doing will bring about a profound and irreversible change in the way we perceive ourselves.

It may seem unusual to write a review article on an activity that has yet to produce positive results, at least as they relate to the principal objective of the activity. However, as hopefully will be clear, the continuing failure to detect other planetary systems has significant implications for other problems such as the mass function at the low end of the main sequence. Further, the detection of planetary-mass companions to the millisecond pulsar PSR1257+12(Wolszczan \& Frail 1992) raises important questions regarding the relationship of such systems to planetary systems; those questions are discussed later in the paper.

The currently accepted paradigm for the formation of stars and planetary systems is outlined in Section 2, leading to a discussion of the underlying 
scientific rationale for and objectives of a comprehensive effort to search for and characterize other planetary systems. Highlights from search efforts to date are presented in Section 3, with emphasis on results that have been obtained within the past decade. The scientific significance of null results from searches to date, along with a focused discussion of the exciting discovery of planetarymass companions to the millisecond pulsar PSR1257+12, are presented in Section 4; it is shown in this section that this particular discovery is relevant, but not equivalent, to the scientific objectives framed here. Section 5 provides a brief, and certainly incomplete, glimpse of future prospects in this emerging field of research along with some concluding remarks.

\section{THE PARADIGM BRIEFLY STATED}

The central focus of this article is the status of searches for other planetary systems. However, there will arise interpretive issues associated with results that have been reported in the literature, as well as a need to establish a common basis for interpreting future observations. This section provides a synopsis of the current paradigm for understanding the origin of the Solar System.

\section{General Features of Our Planetary System}

It is not surprising that efforts to understand the origin of our planetary system derive in no small way from the features of that system. The extent to which any of these features are prototypical of planetary systems in general is unknown at present. Keeping this rather significant caveat in mind, we ask: What are the overarching features of the Solar System that require explanation by any model or theory of the origin of the system?

Those features cited most often are, in no special order, angular momentum and mass distribution, orbital architecture, and planetary composition and obliquity.

ANGULAR MOMENTUM AND MASS DISTRIBUTION The angular momentum of the Sun is $1.6 \times 10^{48} \mathrm{~g} \mathrm{~cm}^{2} \mathrm{~s}^{-1}$, which is due mainly to its spin, whereas the angular momentum contained in the planets, due mainly to their orbital motion about the barycenter of the Solar System, is $3.1 \times 10^{50} \mathrm{~g} \mathrm{~cm}^{2} \mathrm{~s}^{-1}$. That is, the Sun contains less than $2 \%$ of the total angular momentum in the planetary system. In contrast, the total mass of the planets is $1.3 \times 10^{-3} M_{\odot}$; thus, the Sun contains some $99.8 \%$ of the mass in the Solar System.

ORBITAL ARCHITECTURE There are several facets of the general orbital structure of the Solar System that are noteworthy. The orbits of the planets are nearly coplanar; the notable exceptions to this are the innermost (Mercury) and outermost (Pluto) members of the system. Specifically, the orbits are within four degrees of the ecliptic plane. The invariable plane of the Solar System is nearly coincident, within about $7^{\circ}$, with the equatorial plane of the Sun. The 
significance of this offset between the solar equatorial plane and the invariable plane of the planetary system is unclear, and it is generally ignored by modelers of Solar System formation. Planetary orbits are also nearly circular, or at least have very low eccentricity. Orbital eccentricities range from 0.0068 for Venus to 0.0934 for Mars. Mercury $(e=0.206)$ and Pluto $(e=0.25)$ are significant departures from this regularity. All of the planets revolve about the barycenter of the planetary system in a direct sense, i.e. in the same sense as the spin of the Sun. Finally, there is an overall regularity in the spacing of the planets, first noticed independently by Titius and by Bode and generally referred to as Titius-Bode Law. While this spacing has several semi-empirical forms and is accorded widely ranging significance by modelers, it is the case that the relative spacing between planets increases systematically with increasing distance from the Sun. It has been shown by Gladman \& Duncan (1990) that the regions between the present planets are unstable to perturbations by the planets on time scales shorter than the age of the Solar System.

PLANETARY COMPOSITION AND OBLIQUITY The planets fall into three broad compositional categories: the principally rocky terrestrial planets (Mercury, Venus, Earth, and Mars), the gas giants (Jupiter and Saturn), and the icy planets (Uranus and Neptune). The terrestrial planets are located in the inner regions of the Solar System (within 1.5 AU) and have bulk compositions dominated by dense, refractory (high condensation temperature) elements. The gas giants are next in the spatial progression away from the Sun and are typified by low mean densities and compositions that are dominated by hydrogen and helium, although even in the case of Jupiter, the relative abundance of hydrogen and helium to heavy elements is lower than it is in the Sun. The distant icy planets have uncompressed densities that are intermediate between those of the terrestrial and gas giant planets. It is not known with certainty whether the high-Z compositional component that is present in these outer planets is dense rocky material or melted ices of $\mathrm{H}_{2} \mathrm{O}, \mathrm{NH}_{3}$, and $\mathrm{CH}_{4}$ (Lissauer 1993). It should be noted that the masses of the planets also correlate with these three general compositional categories. The Earth is the most massive of the terrestrial planets, whereas the gas giants are 2-2.5 orders of magnitude more massive, and the icy planets are roughly a factor of 10 more massive, than the Earth.

\section{The Paradigm}

The current paradigm for the formation of the Solar System has its evolutionary roots in the notions of Kant and Laplace in the middle to late eighteenth century and is referred to generally as the nebular hypothesis. The defining attribute of the paradigm in its current state of evolution is that the process of star formation involves a disk associated with a stellar embryo. The embryo grows through accretion of material out of the disk, and planetary companions are formed from the same disk. The key for the purpose of this article is that the planetary 
system is formed from a structure that should, at least in a qualitative sense, accompany every star that is formed. Its formation thus provides a fossil record of events and conditions at that time in the evolution of the star. The real issue then is whether the quantitative aspects of such disks lead inevitably to the formation of a retinue of planetary companions to those stars, that is, a planetary system, or whether that outcome is but one of many, and perhaps one with a low probability of occurrence.

Excellent reviews of all aspects of star and planetary system formation can be found in Protostars and Planets II (Black \& Matthews 1985) and III (Levy \& Lunine 1993). Interested readers should consult those volumes for specific references on detailed aspects of the material to follow.

The basic notion is that the process of star formation is initiated through gravitational collapse in slowly rotating molecular cloud cores. It is thought that during the very early stages of collapse, magnetic fields present in the collapsing core will tend to maintain a constant angular velocity as the core becomes smaller in spatial extent. At some point, either through weakening or loss of magnetic field through ambipolar diffusion, the collapsing core becomes decoupled from the general background and collapse proceeds at nearly constant angular momentum. Such a collapse quickly leads to a strong enhancement of rotational forces, giving rise to a configuration that is highly flattened, i.e. a disk or nebula. The details of this process-for example, the spatial extent of the resultant disk-depend upon many factors and is an issue of debate among students of the subject. It is envisioned that the process of planet formation from the disk proceeds through a number of steps, but the underlying general mechanism is that of accretion. [See Lissauer (1993) for a recent review of planet formation.]

Planets appear to be the end result of a bottom-up assembly process, beginning with the accumulation of essentially interstellar grains into millimeter- to centimeter-size objects that are formed as dust settles to the midplane of the disk. These larger objects are in turn brought together to form kilometer-scale objects; these planetessimals proceed through what is known as runaway accretion to form bodies of lunar size. The final stage of the process, at least for the terrestrial planets, is the accumulation of these lunar-size bodies into planets as we know them. A signature of this process is a violent and random series of events, with collisions between objects of comparable size during the final stages of the process. Current thinking is that the Moon is the result of a collision between the young Earth and a Mars-size object. Such collisions can clearly have a significant effect on, among other things, the obliquity of the planet.

The picture is somewhat different in the case of the giant and icy planets. In the regions of the disk where it is presumed that these planets would form, the idea is that a planetary core would accrete until a critical mass of around 
10 Earth masses is reached (Mizuno et al 1978). At that point, the growing core in the presence of substantial amounts of gas (the nebular gas in which the growing core is embedded) is no longer able to sustain an equilibrium atmosphere, and a very rapid accretion of nebular gas occurs, which gives rise to a giant planet.

There is increasing observational evidence that at least the early phases of the paradigm are correct. Millimeter-wavelength observations of regions where stars are forming indicate the presence of disklike structures. Much of this evidence is inferential in nature. Observations of $\mathrm{CO}$ and other molecules in regions near forming or young stars show evidence of highly collimated, bipolar flows centered on those stars. These same objects appear to have high-density, small-scale structures centered on the stars that are oriented at right angles to the bipolar flow. These structures are generally interpreted as circumstellar disks with the bipolar flow being channeled out from the poles, which are usually presumed to coincide with the rotation axis of the central object.

While the evidence that the early stages of star formation involve a disk is growing, there is as yet no firm evidence to reveal the fate of the disk material. There are three general fates of interest: 1 . The material dissipates after feeding the stellar embryo and nothing of consequence is formed from the disk; 2 . two stars, or a star and a brown dwarf, are formed; and 3. a planetary system is formed. Recent observational studies (Duquennoy \& Mayor 1991) suggest that as much as $64 \%$ of stars are members of binary systems; other studies derive an ever greater value, with the fraction of single stars being no more than 5\% (Poveda 1988). This would suggest that the fate of most disks seen in association with pre-main-sequence stars is the formation of two stars. There have been reports of structure in some disks that has been attributed to gaps in the disk caused by clearing of material as planets are or have been accreted (e.g. Marsh \& Mahoney 1992). This is an interesting conjecture, but it is not the only reasonable interpretation of these observations. The data could be a consequence of the opacity structure of the disk (Boss \& Yorke 1993) rather than a physical gap.

\section{Consequences of the Paradigm}

During the two centuries following Laplace and Kant, most concepts regarding the origin of the Solar System were just that, concepts. They were not quantifiable theoretical constructs that could be tested by observation. Perhaps the most significant aspects of the current paradigm are that it is quantifiable to a large degree and that it makes rather general predictions about the frequency of occurrence and the nature of planetary systems.

If the paradigm is substantially correct, planetary systems should be found in association with most if not all single stars. This deceptively simple consequence of the paradigm cannot be overemphasized. It has profound implications for observational astronomy and for the way in which humanity perceives itself. 
Our current understanding of how binary stars form is too rudimentary to permit an equivalent statement regarding the frequency of occurrence of planetary systems in association with this most prevalent form of stellar systems.

The paradigm would also suggest that the general architecture of planetary systems should be similar to that of our own system, e.g. roughly coplanar, circular orbits and some form of systematic compositional gradient in the bulk composition of the planets that in a very broad form would reflect the temperature and density structure of the disk from which the planets formed. One would expect, for example, that any gas giants would be located in the outer regions of the system where the temperature is cool enough for the abundant volatile species (e.g. water) to be present in solid form.

\section{Scientific Rationale and Objectives of Searches}

What then is the underlying scientific rationale for placing substantial effort and resources into searches for other planetary systems? There are three that come to mind. Prominent among the three is that the results from a comprehensive search for other planetary systems are essential if we are to ever understand the origin of our own planetary system. While we can gain insight into the conditions and processes that were operative during the formation of the Solar System by studying the Solar System and its components, the only way that we can test our model scientifically is through the study of other examples of the phenomenon.

A second element of the rationale is found in the causal relationship between star formation and the formation of planetary systems. If the paradigm described here is correct in its broad outlines, then as noted above, planetary systems should accompany virtually every single star. This assumes something about the likely evolutionary path of the disks now seen in association with forming or young stars. If that assumption is incorrect-if the dynamical evolution of the disk does not lead inevitably to the formation of a planetary system-then our model of the star formation process may need some modification. If planetary systems are found to be rare, it is likely that the basic tenets of our current theory of star formation are unfounded. Conversely, if planetary systems occur as predicted in great abundance, details of their architecture and composition will provide unique long-lived fossil tracers of the dynamics of star-forming disks.

Finally, there is the other currently unique observational aspect of the Solar System: life. Planets can be thought of as cosmic petri dishes. They provide the essentials for life to begin and evolve. If life is to be found outside of the Solar System, particularly highly evolved life, planetary systems will likely be required. While detection of planetary systems does not of itself prove that extraterrestrial life exists, it does provide support for the contention that life could arise elsewhere. It also provides specific targets for any concentrated effort to search for signs of life beyond the Solar System. 
Given the rationale above, what are the specific scientific objectives of a search effort? These are listed below in roughly the order of increasing observational complexity.

- Detection of another planetary system. The confirmed detection of another planetary system is the first scientific objective of any focused search effort.

- Establishing the statistical nature of planetary systems. While the confirmed detection of another planetary system will be very exciting and will no doubt provide cover material for popular news magazines, the real scientific return from searches begins when we gather statistical information on planetary systems. For example, what is their frequency of occurrence? Are they found only in association with single stars, or are they present in association with binary systems? Are they found in association with all spectral types, or do they prefer stars like the Sun? It will be necessary to search a large number of stars in a comprehensive way if we are to address this objective.

- Detailed study of individual planetary systems. Once planetary systems are located, it follows that we will study aspects of individual members of those systems in some detail. Spectral analysis of the atmospheres of planets revolving around other stars would provide a measure of compositional trends in the system, but it also could provide insight into details of the planet itself. A particularly intriguing possibility is one similar to the Earth. Anyone with knowledge of chemistry could determine that the Earth's atmosphere is not in chemical equilibrium; the principal cause for disequilibrium is life. Additional details about that life can be gleaned from specifics regarding the composition of the atmosphere. Given adequate spatial resolution and collecting area, one could image an Earth-like planet revolving around another star (see Section 5 for more on this point).

\section{RESULTS TO DATE}

\section{Brief Summary of Search Techniques}

Prior to a discussion of results from searches, it is useful to review briefly the various types of techniques for searching. An extensive discussion of these is given elsewhere (Black 1980a,b).

Search techniques can be placed into two general categories: indirect and direct. The former detect planetary companions to stars by virtue of some observable effect that those companions have on their central star. The latter detect planetary companions by sensing radiation, thermal or nonthermal, that comes from the companions themselves. Indirect techniques focus on observing stars, whereas the direct techniques do everything possible to eliminate interference from the star. 
INDIRECT The three most widely used or considered indirect detection techniques are astrometry, spectroscopy (radial velocity), and photometry. The former two sense the motion of a star around the barycenter of the star-planetary companion system. Astrometry is concerned with the projection of the star's orbital motion on the plane of the sky, and spectroscopy is concerned with the line-of-sight component of that motion. Photometry measures the apparent dimming in the brightness of a star as it is transited by a planetary companion.

If a star has a planetary companion, the center of mass (barycenter) of the star-planet system is offset from the center of mass of the star. This leads to the star revolving about the barycenter with a period equal to that of the planet. The apparent amplitude $\theta$ of an astrometric signal due to the presence of a planetary companion of mass $m_{\mathrm{p}}$ revolving around a star of mass $M$ in a circular orbit of semimajor axis $r$, and with the star located at a distance $D$ from the observer, is given by

$$
\theta=\left(\frac{m_{\mathrm{p}}}{M}\right)\left(\frac{r}{D}\right)=\left(\frac{m_{\mathrm{p}}}{D}\right)\left(\frac{p}{M}\right)^{2 / 3},
$$

where $\theta$ is in arcseconds, $m_{\mathrm{p}}$ and $M$ are in solar masses, $r$ is in AU, $D$ is in parsecs, and the orbital period $P$ is in years. The radial velocity $v$ associated with such a system is given by

$$
v=\frac{30 m_{\mathrm{p}} \sin i}{(r M)^{1 / 2}}=\frac{30 m_{\mathrm{p}} \sin i}{M^{2 / 3} P^{1 / 3}},
$$

where the velocity is in $\mathrm{km} \mathrm{s}^{-1}$ and the other symbols and their respective units are as shown.for Equation 1. The angle of inclination between the line of sight to the star and the normal to the orbital plane of the star-companion pair is denoted by $i$.

It will be useful to have some idea of the magnitude of likely signals for candidate systems of interest. If we consider a planet of mass similar to Jupiter, $m_{\mathrm{p}}=0.001$, located at $5.2 \mathrm{AU}$ from a solar mass star that is a distance of ten parsecs away, then $\theta=0.5$ milliarcseconds. Comparable numbers for an icy planet and for a large terrestrial planet, both located at 5.2 AU, are $\theta=24$ and 1.6 microarcseconds, respectively. While these representative numbers can be higher or lower depending on the assumed values for $M, r$, and $D$, it is clear that detection is a demanding task. Current ground-based astrometric studies using very good systems are accurate to roughly a milliarcsecond, and this performance is also representative of the performance of the HIPPARCOS and Hubble Space Telescope astrometric space systems.

The maximum velocity signal associated with the model system described above is $v=13 \mathrm{~m} \mathrm{~s}^{-1}$. The corresponding values for an icy and terrestrial planet, again located 5.2 AU from the central star, are $v=0.6 \mathrm{~m} \mathrm{~s}^{-1}$ and $6 \mathrm{~cm} \mathrm{~s}^{-1}$ ! While much of stellar spectroscopy today is still done at the level of accuracy of $1000 \mathrm{~m} \mathrm{~s}^{-1}$, there are now a number of groups with systems that 
are capable of radial velocity measurements accurate to better than $10 \mathrm{~m} \mathrm{~s}^{-1}$. We discuss these in more detail below.

Photometric detection of other planetary systems involves detecting an apparent dimming in a star's brightness caused by the physical blocking of light by the transit of a planetary companion to the star between the observer and the star. The magnitude of the apparent dimming is simply the ratio of the transiting planet's area to the area of the star, i.e. $\Delta m_{v}=\left(d_{\mathrm{p}} / d\right)^{2}$, where $d_{\mathrm{p}}$ and $d$ are the diameters of the planet and star, respectively. For a gas giant planet revolving around a solar-type star, $\Delta m_{v}=0.01$ magnitudes. The corresponding values for an icy and terrestrial planet are $\Delta m_{v}=0.001$ and $8.4 \times 10^{-5}$ magnitudes, respectively. The level of performance of relatively routine photometric studies is sufficient to detect the dimming due to transit of the example Jupiter-Sun system, but the other two examples begin to stretch the capability of existing systems.

So far, there have been very few searches using this technique. This is partly due to the need to study a large number of stars $\left(\sim 10^{3}\right)$ because of the probability that the Earth lies essentially in the orbital plane of any planetary system associated with an arbitrarily selected star. However, some groups are developing concepts for both ground- and space-based instruments that could undertake searches using this method and some innovative observational choices (e.g. eclipsing binaries) or technology (e.g. Borucki \& Summers 1984, Tutukov 1987).

One possible detection method not discussed in my earlier review (Black 1980a) is that of gravitational microlensing. This method is outlined here for completeness. It is not, for reasons given below, a particularly useful search method.

The underlying phenomenon for this method is the bending of light that occurs when light passes sufficiently close to a massive object that lies between the source of light and an observer. If the source and the deflecting mass are nearly collinear with the observer, a double image of the source would appear if the observing instrument had infinite spatial resolution.

If we consider the case of stars in the Galaxy (and therefore typically located, on average, several kiloparsecs away from a solar-mass star located between the distant stars and an observer on the Earth), the lensing-event cross section is of order a milliarcsecond in scale. As this is far smaller than the resolution of existing telescopes, the two images would overlap, and an observer would perceive a brightening in the target star during the lensing event. The amplitude of the brightening varies inversely with the impact parameter of the lensing event. Given typical relative motions of a few hundred kilometers per second in the Galaxy, the duration of the lensing event for a solar-mass lens would be of order a month (the duration scales as the square root of the lensing mass).

One way to implement this technique is to observe dense stellar fields through intervening dense fields - Galactic bulge stars through the Galactic disk for 
example. If the lensing object is a binary, as most stars are, the lensing-event light curve will be modified from the simple shape that would be expected for a single star. It has been argued (Gould 1992) that one can observe effects if Jupiter-size objects associated with lensing stars are preferentially located at the ice-condensation zones associated with those stars.

As innovative as this scheme is, it remains to be shown that data can be gathered that will admit such a clear interpretation of the observations in terms of planetary systems. Moreover, the transitory nature of the event makes it difficult to corroborate by independent observers, and it affords no useful diagnostic data concerning the planetary systems that might be discovered. Because this technique does not address the scientific objectives indicated above, I do not view it as significant in the menu of search techniques.

DIRECT Relative to stars, planets are poor emitters of radiation, be it thermal or nonthermal. This is because planets are small in comparison to stars and they are much cooler (the energy emitted in the form of blackbody radiation scales as $R^{2} T^{4}$, where $R$ is the radius and $T$ is the temperature of the emitting body).

The relative brightness of a planet and star depends on the observed wavelength as well as on the physical parameters of the planet and star pair (e.g. their relative sizes and separation). Viewed at optical wavelengths, that is in reflected light, Jupiter would appear to be roughly a billion times dimmer than the Sun if viewed from a distant perspective. Viewed in the thermal infrared at say $\lambda=30 \mu \mathrm{m}$, Jupiter would only be about four orders of magnitude less bright than is the Sun. Consider also that when viewed from a distance of ten parsecs, Jupiter's maximum angular separation from the Sun is 0.5 arcseconds. The relative fluxes as a function of wavelength for the Sun, Jupiter, Neptune, and the Earth are shown in Figure 1.

As a matter of spatial resolution in the Raleigh sense, i.e. resolving objects of comparable brightness, 0.5 arcsecond separation is not daunting (the resolution of the Hubble Space Telescope is roughly a factor of ten better than this value). However, it is the combination of angular resolution and brightness difference that makes direct detection such a challenge because of scattered light, among other effects. A thorough discussion of direct detection is given elsewhere (Black 1980a).

\section{Search Results Since 1980}

Most of the searches to date have involved indirect detection techniques, principally those of astrometry and spectroscopy. One of the better known astrometric studies, which involved data taken over much of this century, is that by van de Kamp over many years of observing at the Sproul Observatory (e.g. van de Kamp 1969). What has made this study so well known is that van de Kamp concluded that Barnard's star had not one, but two, Jupiter-mass companions in orbit with periods of 12 and 26 years. Given the proximity of Barnard's 


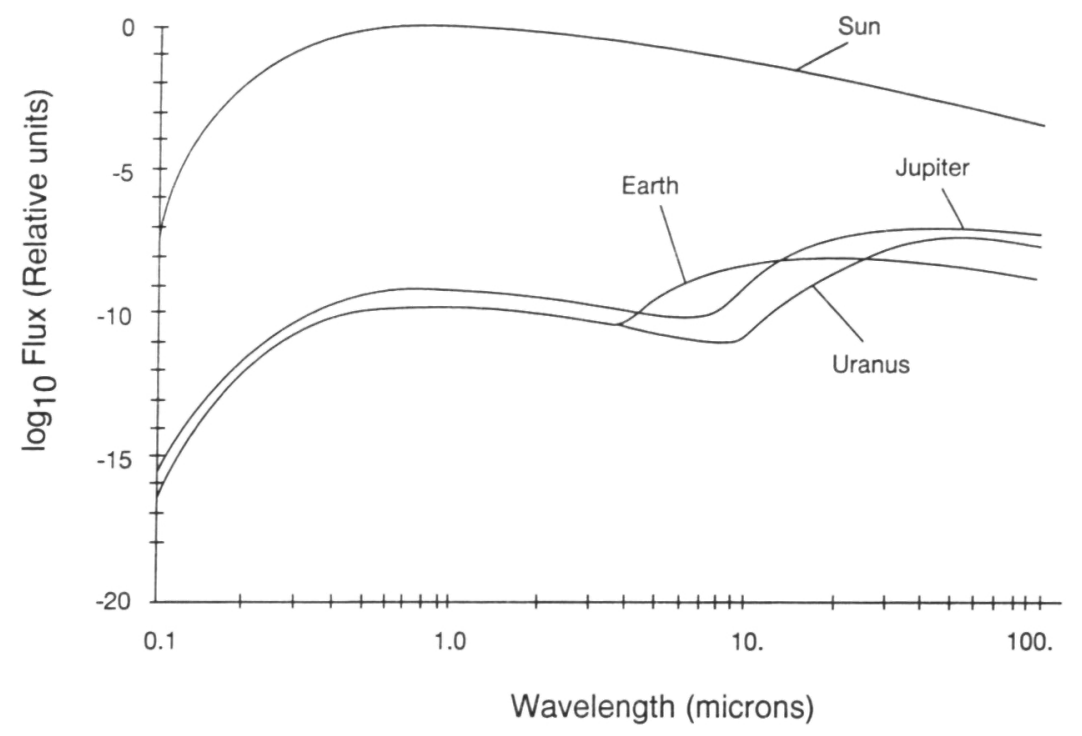

Figure 1 The relative spectral energy distribution of representatives of the three classes of planets: terrestrial (Earth), gas giant (Jupiter), and icy (Uranus). Shown for comparison is the spectral energy distribution from the Sun. The curves are normalized so that the Sun's peak flux is defined as unity.

star to the Sun (1.8 parsecs), this interpretation if true would seem encouraging for those who felt that planetary systems occur frequently in nature and that they would all be similar in a general sense to our own. However, independent and more accurate studies of Barnard's star by Gatewood and coworkers (e.g. Gatewood \& Eichhorn 1973; GD Gatewood, personal communication 1994) and by Harrington and coworkers (e.g. Harrington \& Dahn 1980) have failed to confirm van de Kamp's interpretation. The absence of any detection in the Allegheney Observatory data is now placing stringent constraints on the nature of companions to Barnard's star (Figure 2).

Harrington et al (1983) reported evidence of a perturbation in the motion of the star Van Biesbroeck 8, (VB 8). His early efforts to characterize the source of this perturbation suggested that it was an object with an orbital period of decades and a mass in the range 0.02 to $0.08 M_{\odot}$. An independent discovery of a companion to this star was made using infrared speckle observations by McCarthy et al (1985). These observations were sufficiently exciting that a conference was held (Kafatos et al 1986) to discuss brown dwarfs with emphasis on VB $8 \mathrm{~b}$, the companion. There was considerable debate over the mass of the companion, and the conclusion that emerged from the various theoretical studies was that VB $8 \mathrm{~b}$ was probably about 50 times the mass of Jupiter. Unfortunately, all efforts to study this companion further have failed to even detect it. It seems that VB 8b either does not exist or is hiding from us. 
I mention the cases of Barnard's star and VB $8 \mathrm{~b}$ because they serve as warnings that the search is a very demanding observational task-one that has been at the very limits of the capabilities of observational techniques. There is a very high mortality rate for claimed detections of other planetary systems.

One could easily number the serious searches for other planetary systems prior to 1980 on the fingers of two hands. Fortunately, that has changed significantly in the past decade owing mainly to the use of radial velocity and direct detection techniques. However, the number of sufficiently accurate astrometric programs remains small.

Eight groups have ongoing radial velocity efforts to search for nonstellar companions to other stars. Three of these studies were conducted with moderate accuracy (e.g. $\sigma>100 \mathrm{~m} \mathrm{~s}^{-1}$ ), while the other five were at relatively high accuracy $\left(\sigma \lesssim 50 \mathrm{~m} \mathrm{~s}^{-1}\right.$ ). DW Latham and colleagues (Latham 1992, 1994 personal communication) have been conducting a search of roughly a hundred stars over a ten-year time span. The level of accuracy of their search is about $500 \mathrm{~m} \mathrm{~s}^{-1}$. An eight-year study of 540 stars, with an accuracy of $300 \mathrm{~m} \mathrm{~s}^{-1}$ has been conducted by Duquennoy \& Mayor (1991; also Mayor et al 1992). Marcy \& Benitz (1989) examined 65 stars over a four-year interval with an accuracy of $230 \mathrm{~m} \mathrm{~s}^{-1}$.

The shortest time base of the eight studies is three years (Murdoch et al 1993); this study has an accuracy of $50 \mathrm{~m} \mathrm{~s}^{-1}$ and includes 29 stars. Four of the studies

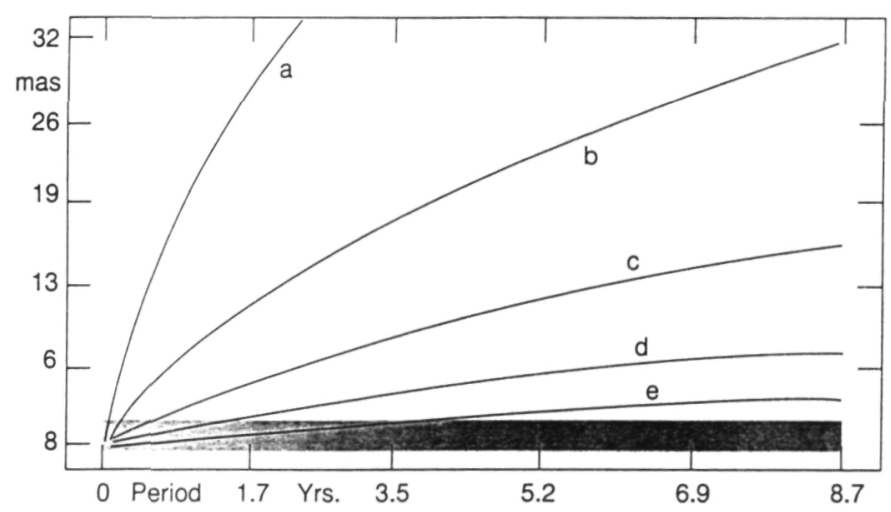

Figure 2 A family of expected perturbations in the motion of Barnard's star (in milliarcsecondsmas) for companions of various masses. The curves labeled a-e correspond, respectively, to $m_{\mathrm{p}}$ values of $10,4,2,1$, and 0.5 Jupiter masses. The expected perturbation is that described by Equation 1 with the orbital period displayed on the abscissa expressed in years. The shaded area is a measure of the uncertainty in the Allegheney observations. Any signal above this region would have been detected (e.g. any companion with mass greater than four Jupiter masses would have been detected unless its orbital period is either less than about 0.2 years or longer than the observing program, roughly 8.7 years; similarly, a companion with mass of 0.5 Jupiter masses would have been detected if its period fell in the range of roughly 4 to 8.7 years). This figure is courtesy of G Gatewood. 
have been conducted with $\sigma=15 \mathrm{~m} \mathrm{~s}^{-1}$. The longest such study, 12 years, is that by Campbell and Walker of 21 stars (e.g. Campbell et al 1988, Walker et al 1994). The remaining three have been ongoing for seven years and have involved 20 stars (Marcy \& Butler 1992), 13 stars (McMillan et al 1994), and 30 stars (Cochran \& Hatzes 1994).

The searches by Duquennoy \& Mayor led to nine cases where the inferred $m_{\mathrm{p}} \sin i$ value was $\leq 0.08 M_{\odot}$; that is, possible cases of brown dwarf companions. Latham and coworkers found two examples where the inferred $m_{\mathrm{p}} \sin i$ value was $0.08 M_{\odot}$. Interestingly, no similar values of $m_{\mathrm{p}} \sin i$ have yet resulted from the suite of searches with high accuracy. We return to this apparent discrepancy below.

One of the stars studied by Latham and coworkers, a radial velocity standard, is HD 114762. This F9V star showed clear evidence for a perturbation with a periodicity of 89 days (Latham et al 1989). These values, along with a mass for the primary of $\approx 1.1$ solar masses, gives a value for $m_{\mathrm{p}} \sin i \approx 11$ Jupiter masses. Independent observations of HD 114762 by Cochran et al (1991), using the more accurate system located at the McDonald Observatory, confirm the general perturbation detected by Latham and coworkers (Figure 3). Cochran et al's

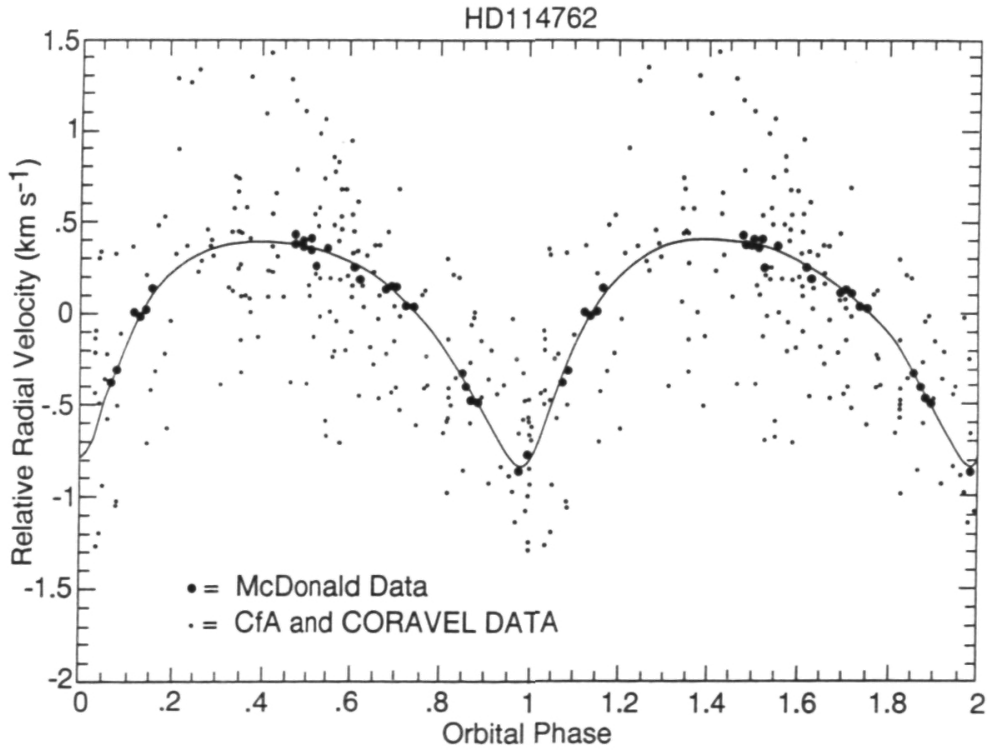

Figure 3 The radial velocity curve for HD114762 shown as a function of orbital phase. The smaller dots are the data presented by Latham et al (1989) and includes observations by the Center for Astrophysics and the CORAVEL (COrrelation RAdial VELocities) instruments. The larger dots are the data from the high-accuracy radial velocity study by Cochran et al (1991). The solid line is the orbital solution from the McDonald Observatory study. 
observations do, however, indicate that the orbit of the companion has a relatively high eccentricity $(e \approx 0.38)$, consistent with what is observed for binary stellar systems, but high in comparison with planetary orbital eccentricities.

Cochran also notes that the rotational velocity of $\mathrm{HD} 114762, \approx 0 \mathrm{~km} \mathrm{~s}^{-1}$, is anomolously low for a main sequence star of its spectral type. He argues that this is likely to be due to the fact that we happen to be viewing this star from its rotational pole, i.e. $\sin i \approx 0$. If one assumes that the orbital plane of any companion(s) to HD 114762 lie in or near to the equatorial plane of the star, then the likely companion mass to this star is much higher than its minimum mass value of $\approx 11$ Jupiter masses. Cochran et al conclude that the companion is most likely to be a star near the bottom of the main sequence.

The most accurate astrometric study is that by Gatewood and collaborators (GD Gatewood, personal communication 1994). The current high-accuracy program at Allegheney involves 21 stars and has been ongoing for seven years. This study has not revealed the presence of any companions with $m_{\mathrm{p}} \leq 0.08 M_{\odot}$.

The searches mentioned above all use indirect search methods. There have been a number of search efforts using direct techniques, most of them in the infrared and most of them focusing on the issue of low-mass/brown dwarf companions to $\mathrm{M}$ dwarf stars as well as to young stellar objects (e.g. T Tauri stars). A summary of these observations, as well as a comparison of the direct search for companions with a subset of the radial velocity searches mentioned above, has been given recently by Heacox \& Gathright (1994). Readers are referred to that paper for references to the specific searches.

The major finding in the Heacox \& Gathright work as it relates to the subject of this review is that results from two direct searches for companions to $\mathrm{M}$ dwarfs seem to be at odds with one another, and at least one way to reconcile the differences leads to a discrepancy with the findings of Fischer \& Marcy (1992). Most of these searches involve a statistically marginal number of objects, so any discrepancies at this stage may be more apparent than real. It would be helpful if objects that are purported to have companions could then be studied extensively by other techniques or groups to corroborate the existence of the companion. One thing is clear: Although these searches and others have provided an excellent characterization of the low-mass end of the main sequence, they have as yet provided no detection of a planetary system.

\section{A FUNNY THING HAPPENED ON THE WAY TO THE FORUM}

\section{Wither the Brown Dwarfs?}

It is often the case that scientific searches are expressed in terms of a pursuit of the Holy Grail or a search for the Rosetta Stone. It seems to me that, at least at the present time, there is a more apt analogy for our efforts to find other 
planetary systems. There exists in American movie cartoons a well-known coyote character who is constantly, and unsuccessfully, in pursuit of a road runner. The image that often comes to mind as the coyote frantically chases his fast and illusive prey is one of the coyote so focused on the road runner that he fails to sense that he is no longer running on a path, but is instead suspended in the air over a deep ravine into which he inevitably plunges.

Just at the coyote is focused on his objective and fails to recognize that he is no longer on solid ground, many researchers are focused on detecting another planetary system and in so doing they have not appreciated that their failure to discover few, if any, signals could be of great significance for our understanding of another class of objects, brown dwarfs. While the failure to detect other planetary systems is not yet statistically significant in terms of the existence of such systems, the paucity of apparent brown dwarf detections is becoming so. The discussion that follows is based on a thought-provoking paper by Marcy \& Butler (1994) concerning the results from precise radial velocity searches for other planetary systems.

It is useful to recast Equation 2 as follows

$$
m_{\mathrm{p}} \sin i=0.033 v M^{2 / 3} P^{1 / 3} \text {. }
$$

As a consequence of the $\sin i$ term, radial velocity surveys are inherently statistical in nature. If one wants to know the expected number of $m_{\mathrm{p}} \sin i$ outcomes of a particular value from a survey of $N$ stars, one needs to specify the distribution of period $P$ and of secondary masses $m_{\mathrm{p}}$. The usual assumption is that systems are randomly oriented with respect to the line of sight for an arbitrary observer.

Marcy \& Butler have examined all of the stars studied in the eight radial velocity surveys mentioned above in an effort to constrain the extent to which the observations set meaningful statistical limits on the occurrence of brown dwarfs and planets. They assume the same Gaussian distribution in $\log P$ that was obtained by Duquennoy \& Mayor, and they tested various assumed distributions for secondary masses, ranging from one that is flat for values of secondary masses above the hydrogen-burning limit of 0.08 solar masses, but truncated to zero for lower masses, to ones that would imply an increasing number of objects as the mass decreases for masses above the hydrogenburning cutoff, again with abrupt truncation. The outcome of their calculations are histograms of expected values of $m_{\mathrm{p}} \sin i$ from the actual survey population.

They found for the case of a flat mass distribution with a cutoff that one would expect to observe four cases of $m_{\mathrm{p}} \sin i \leq 0.08 M_{\odot}$ from the survey of 540 stars by Duquennoy \& Mayor; this is to be compared with the nine that they detected. This suggests that five of these nine are legitimate brown dwarf candidates, given the assumption about the mass spectrum. The four impostors in their results are simply low-mass stars with relatively high inclinations $(\geq 60$ 
degrees) to the line of sight. The statistical uncertainty associated with the analysis is such that as many as six of the nine could be impostors, but that still would leave three candidates.

Note that when Marcy \& Butler assumed a very mild power law for the mass distribution, i.e. $N(M)=c M^{-0.8}$, again with an assumed hard truncation at the nuclear burning cutoff, all nine of the brown dwarf candidates in the Duquennoy $\&$ Mayor survey could be accounted for with no real brown dwarfs. This assumed power law is flatter than that found by Duquennoy \& Mayor (index of -1.3 ), and it is much flatter than the Salpeter mass spectrum (index of -2.35).

A similar analysis of the Latham survey population suggests that one expects to obtain one brown dwarf candidate from their sample of 100 stars if the flat mass distribution is assumed; they find two [the difference, while small, is due again to inclination effects, consistent with the suggestion by Cochran et al (1991) for the star HD 114762]. As with the larger survey by Duquennoy \& Mayor, this survey yields no evidence for brown dwarfs if there is even a slight power law for the mass function as defined above.

One might wonder why the far more accurate radial velocity searches show no cases where $m_{\mathrm{p}} \sin i \leq 0.08 M_{\odot}$. The reason appears to be that the surveys with accurate systems have examined too few stars. A sample size of order 50 is needed to test statistically the findings of the less accurate systems.

It would be useful to have more studies of the type conducted on HD 114762, in which a high-accuracy, radial velocity system is used to study one of the candidate brown dwarfs that have been discovered by a lower-accuracy survey.

Marcy \& Butler conclude that an upper limit of $1 \%$ can be established for the frequency of brown dwarf companions revolving about spectral type $\mathrm{F}$ through $\mathrm{M}$ main-sequence primaries in orbits of $4 \mathrm{AU}$ or less. They also conclude that low-mass brown dwarfs (10 to 40 Jupiter masses) exist around no more than 5\% of $\mathrm{G}$ and $\mathrm{K}$ dwarfs in orbits of $5 \mathrm{AU}$ or less. A final conclusion by these authors, using data from their own high-accuracy studies of 20 stars, is that even the mildly increasing mass function (index of -0.8 ) can be shown not to extend to masses below $0.08 M_{\odot}$. Their data are insufficient to rule out the possibility of a flat mass function in the brown dwarf range; more stars are needed to establish that constraint.

While the high-accuracy surveys are not yet compelling in terms of the existence of planetary systems, Marcy \& Butler conclude that based on all of the available data, planets with masses greater than 3 Jupiter masses and in orbits of $5 \mathrm{AU}$ or less occur with a frequency of less than $3 \%$.

\section{The PSR $1257+12$ Discovery}

The history of scientific discovery is one of serendipity; Examples abound in all branches of science. One of note in astronomy is the discovery of pulsars in the late 1960s. Researchers were studying interstellar scintillation when they happened upon what we now refer to as pulsars. 
Some of the early efforts to explain the pulsars linked them with extraterrestrial intelligence because of the extraordinary, and until then undetected, regularity in the pulses. That model has faded as we have come to know more about pulsars, but it makes the recent discovery by Wolszczan \& Frail, using time of arrival data, of two planetlike companions to the millisecond pulsar PSR1257 + 12 somewhat ironic.

The Wolszczan \& Frail discovery is not the first time planets have been thought to have been detected in association with pulsars. Shortly after pulsars were detected, Richards et al (1970) reported a possible detection of planets in association with the Crab pulsar. An excellent summary of pulsars and planets can be found in the conference proceedings edited by Phillips et al (1993). Here I focus on some key aspects of the PSR $1257+12$ case.

The putative planetary companions to PSR $1257+12$ have $m_{\mathrm{p}} \sin i$ values of 3.4 and 2.8 Earth masses, and their respective orbital periods about the pulsar are 66.5 and 98.2 days. It was recognized independently by Malhotra et al (1992) and Razio et al (1992) that if the companion interpretation was correct, then one should be able to observe the consequences of the mutual gravitational perturbation of the companions on each others motion within a few years. The predicted perturbations have apparently been confirmed by A Wolszczan (1994, personal communication).

Malhotra et al were able to place constraints on masses of the companions because of the near 3:2 resonance condition of the companion orbits. This placed the masses near the lower end, i.e. $\sin i$ is not small. The evidence is that the orbits are nearly circular $(e=0.02)$ and coplanar.

A discussion of the origin of millisecond pulsars is beyond the scope of this article. It seems clear that the companions were not present when the pulsar progenitor was on the main sequence, and it seems likely that they formed from some disklike structure associated with the pulsar as it was transformed into a millisecond pulsar; PSR1257 +12 is a bit like Picasso: It had these children very late in its life. In this respect, it is significantly different from the Solar System where the formation is during the infancy of the star. The physical (e.g. thermal) and chemical (e.g. presence of volatiles) states of any nebula associated with PSR1257+12 are bound to be remarkably different from those associated with star formation in general and the early solar nebula in particular.

\section{The Family Tree}

Accepting for the moment that the companions to PSR $1257+12$ are of planetary mass, the question is how does this system relate to a planetary system? Before giving my answer to that question, consider the following analogy.

Imagine that you are a senior biology professor from a planet revolving around the star Beta Hydri and you wish to study the species Homo sapien rumored to inhabit the third planet revolving around a G-dwarf located only 6.7 parsecs from your star. Field trips are tedious and so you send one of your 
students with instructions to bring back a few specimens for study, but you are a little lax in providing guidance (good students will figure out what to do) and simply specify that you want him to return with a bipedal, warm-blooded, intelligent creature. Your student lands in Borneo, captures a few specimens of Pongo pygmaeus and promptly heads for home (the planet is fairly primitive by the student's standards).

Depending on what our learned professor is interested in, he might be able to determine a great deal about $H$. sapien by studying $P$. pygmaeus; their genetic material is common at about the $95 \%$ level, but there are many significant attributes of $H$. sapien that cannot be gleaned from any analysis of $P$. pygmaeus. Such is the case with planetary systems and the system discovered by Wolszczan $\&$ Frail.

Although there is a superficial similarity between the pulsar system and the Solar System, just as there is between $P$. pygmaeus and $H$. sapien, the study of one is only marginally related to knowledge of the other. Indeed, there are additional examples of systems that show equivalent levels of similarity to the Solar System, namely the regular satellite systems of the gas giant planets. The Galilean satellites of Jupiter and the inner satellites of Saturn move in coplanar, low eccentricity orbits in the equatorial plane of their parent planet. They evidence a regularity in spacing similar to that found in the Solar System, and they are comparable in mass to planets (Ganymede and Titan are both more massive than Mercury).

It is important to keep a clear focus on the scientific rationale and associated objectives of the efforts needed to complete the Copernican Revolution. A central element of the rationale is to test observationally the paradigm that is at hand regarding the formation of stars and planetary systems. We can not test that paradigm, nor gain the type of information that we need to construct an alternative, should the current one turn out to be flawed, if we do not search for and characterize proper analogues. The focus of our searches must be on planetary systems, not extrasolar planets.

\section{CONCLUDING REMARKS}

As more attention and effort is placed on this scientific endeavor, it is worthwhile to establish or reiterate certain guiding principles. Given the significance of discovery, and more importantly of statistical information regarding planetary systems, it is imperative that observations be corroborated by independent research groups, and even better, by independent search techniques (e.g. an astrometric discovery and a radial velocity corroborative observation).

Another point to stress is that where major new instrument thrusts are being considered, be they on the ground or in space, they should be judged in the light of a significance of a null result. A significant, quantitative test of the paradigm outlined here would be provided from a null result as long as the search 

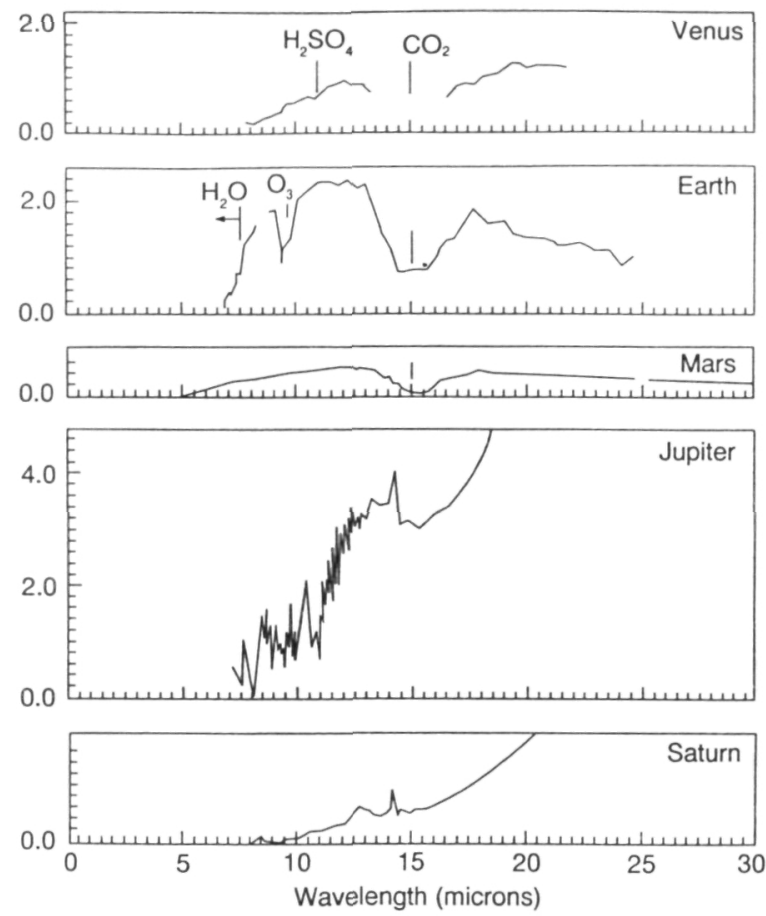

Figure 4 Spectra (in arbitrary units) from three terrestrial planets and two gas giants. Moderateresolution spectra, $\lambda / \Delta \lambda=20-100$, will provide significant information regarding individual planets as well as comparisons among planets.

was capable of detecting objects of roughly the masses of the icy planets, or equivalently, the cores that seem to be required to build gas-giant planets around at least 100 stars. The absence of a Jupiter-like planet would not of itself be a substantial challenge to our paradigm. The absence of objects of masses of the order of 10 Earth masses would cause a major reexamination of our view of the origin of the Solar System and of star formation.

The most difficult observational challenge lies in efforts to study specific planets that have been detected revolving around other stars. As shown in Figure 4, the planets in the Solar System have distinct spectral signatures. With sufficient spectral sensitivity and resolution $(\lambda / \Delta \lambda=20-100)$, it is possible in principle to detect lines due to the presence of ozone, implying the presence of a substantial amount of molecular oxygen. This would require a few hundred square meters of collecting area-the equivalent of 60-100 HST-size aperatures (but not of optical quality). An alternative is to have a large, 16-meter aperture as an element of an interferometric array where the outriggers would be small- 
aperture telescopes to provide spatial resolution. Detection of ozone/oxygen and other diagnostics of life could well provide the final link in the chain of thought that was initiated over 500 years ago by Nikolas Copernicus.

Although this article is intended primarily as a review, we close with a look into the future. It is conceivable that eventually we will have the capability to undertake the daunting task of imaging an Earth-like planet revolving around a nearby star. Placing a $10 \times 10$ array on an Earth-size planet revolving around a star located ten parsecs away would require an interferometric array with a scale of $60 \mathrm{~km}$ if operated at a wavelength of 0.5 microns. The collecting area would need to be significant as well-something of the order of $2 \times 10^{4} \mathrm{~m}^{2}$ (a detail left to the engineers).

We are now clearly embarked upon a journey that will provide within the next decade or so an unambiguous assessment of whether the Solar System is a relatively unique phenomenon or whether, as we and Copernicus would suspect, it is a common occurrence in nature. Either outcome will mark a major step in our efforts to understand the universe in which we live, as well as our place in it.

\section{ACKNOWLEDGMENTS}

This paper is Lunar and Planetary Institute Contribution No. 857. The Lunar and Planetary Institute is operated under Contract No. NASW-4574 with the National Aeronautics and Space Administration.

Any Annual Review chapter, as well as any article cited in an Annual Review chapter, may be purchased from the Annual Reviews Preprints and Reprints service. 1-800-347-8007; 415-259-5017; email: arpr@class.org

\section{Literature Cited}

Black DC. 1980a. Space Sci. Rev. 25:35-81

Black DC. 1980b. Icarus 43:293-301

Black DC, Matthews MS, eds. 1985. Protostars and Planets II. Tucson: Univ. Ariz. Press. $1293 \mathrm{pp}$.

Borucki WJ, Summers AL. 1984. Icarus 58:121

Boss AP, Yorke HW. 1993. Ap. J. 411:L99-102

Campbell B, Walker GAH, Yang S. 1988. Ap. J. 331:902-21

Cochran WD, Hatzes AP. 1994. Astrophys. Space Sci. 212:281

Cochran WD, Hatzes AP, Hancock TJ. 1991. Ap. J. Lett. 380:L35-38

Duquennoy A, Mayor M. 1991. Astron. Astrophys. 248:485

Fischer D, Marcy GW. 1992. Ap. J. 396:178

Gatewood GD, Eichhorn H. 1973. Astron. J. 78:777

Gladman B, Duncan MJ. 1990. Astron. J. 100:1669-75

Gould A. 1992. Ap. J. 392:442-51
Harrington RS, Dahn CC. 1980. Astron. J. $85: 454$

Harrington RS, Kallarakal VV, Dahn CC. 1983. Astron. J. 88:1038

Heacox WD, Gathright J. 1994. Astron. J 108:1101

Kafatos MC, Harrington RS, Maran SP, eds. 1986. Astrophysics of Brown Dwarfs. Cambridge: Cambridge Univ. Press. $276 \mathrm{pp}$.

Latham DW. 1992. In IAU Colloq. No. 135, ed. H McCalister, W Hartkopf, ASP Conf. Ser. 32:110. San Francisco: ASP

Latham DW, Mazeh T, Stefanik RP, Mayor M, Burki G. 1989. Nature 339:38

Levy EH, Lunine JI, eds. 1993. Protostars and Planets III. Tucson: Univ. Ariz. Press. 1596 pp.

Lissauer JJ. 1993. Annu. Rev. Astron. Astrophys. 31:129-74

Malhotra R, Black DC, Eck A, Jackson A. 1992. Nature 355:583-85 
Marcy GW, Benitz KJ. 1989. Ap. J. 344:441-53

Marcy GW, Butler RP. 1992. Publ. Astron. Soc. Pac. 104:270-77

Marcy GW, Butler RP. 1994. In The Bottom of the Main Sequence - and Beyond, ed. CG Tinney. Heidelberg: Springer-Verlag. In press

Marsh KA, Mahoney MJ. 1992. Ap. J. Lett. 395:L115

Mayor M, Duquennoy A, Halbwachs J-L, Mermilliod J-C. 1992. IAU Colloq. No. 135, ed. H McCalister, W Hartkopf, ASP Conf. Ser. 32:73-81. San Francisco: ASP

McCarthy DW. Probst RG, Low FJ. 1985. Ap. J. Lett. 290:L9

McMillan RS, Moore TL, Perry ML, Smith PH. 1994. Astrophys. Space Sci. 212:271

Mizuno H, Nakazawa K, Hayashi C. 1978. Prog.
Theor. Phys. 60:699-710

Murdoch K, Hearnshaw J, Clark D. 1993. Ap. J. 413:349

Phillips JA, Thorsett JE, Kulkarni SR, eds. 1993. Planets Around Pulsars. ASP Conf Ser. Vol. 36. San Francisco: ASP

Poveda A. 1988. Astrophys. Space Sci. 142:67

Razio FA, Nicholson PD, Shaprio SL, Teukolsky SA. 1992. Nature 355:325-26

Richards DW, Pettengill GH, Counselman CC, Rankin JM. 1970. Ap. J. Lett. 160:L1

Tutukov AV. 1987. Sov. Astron. 31:663

van de Kamp P. 1969. Astron. J. 74:757

Walker GAH, Walker AR, Irwin AW, Larson AM, Yang SLS, Richardson DC. 1994. Preprint

Wolszczan A, Frail DA. 1992. Nature 355:145 